\title{
Copper bronze Catalyzed Heck Reaction in Ionic Liquids
}

\author{
Vincenzo Calò, Angelo Nacci, Antonio Monopoli, ${ }^{*}$ Eliana Ieva and Nicola Cioffi
}

\section{Supporting information}

\section{General Remarks}

All the starting materials and ionic liquids were commercially available and were used without further purification. Copper bronze was purchased by BDH. The cross-coupling products were identified by comparison of their physical and spectroscopic data with those reported in the literature.

\section{TEM morphological characterisation}

All the samples were observed at $100 \mathrm{kV}$ under a PHILIPS 400T TEM. Evaluation of the copper nanoparticles' core diameter was performed manually over more than 600 particles, selected in different regions of the micrographs.

\section{Typical procedure for the copper bronze catalyzed Heck reaction.}

To tetrabutylammonium bromide $(3 \mathrm{~g}, 9.3 \mathrm{mmol})$ were added tetrabutylammonium acetate $(0.452 \mathrm{~g}$ $1.5 \mathrm{mmol})$, iodobenzene $(0.204 \mathrm{~g}, 1 \mathrm{mmol})$, butyl acrylate $(0.154 \mathrm{~g}, 1.2 \mathrm{mmol})$ and copper bronze (BDH flakes, 99\% or Aldrich copper bronze 99\%) (1.91 mg, $0.03 \mathrm{mmol}, 3 \%$ ). The reaction was heated at $130{ }^{\circ} \mathrm{C}$ under nitrogen atmosphere with stirring for the proper time. At the end of the reaction and after cooling to r.t., the solid mixture was extracted with cyclohexane $(5 \times 20 \mathrm{ml})$ leaving the catalyst in the ionic liquid that can be recycled. The extracted phases were collected and washed with diluite $\mathrm{HCl}$ to remove tributylamine. After the solvent removal, in vacuo, the mixture was chromatographed on a silica pad affording cinnamates in high purity as shown by ${ }^{1} \mathrm{H}$ and ${ }^{13} \mathrm{C}$ NMR spectra reported below.

Procedure for the catalyst recycling.

After completion of the reaction and after cooling to r.t., the products and the unreacted reagents were extracted with cyclohexane $(5 \times 20 \mathrm{~mL})$. After removal of residual organic solvent under vacuum; the resulting solid mixture, containing both the IL and the catalyst, was charged with fresh reagents and heated at $130{ }^{\circ} \mathrm{C}$ under nitrogen atmosphere with stirring for the proper time. 
Spectral data of cinnamates:

(E)-3-(4-Methoxy-phenyl)-acrylic acid butyl ester. ${ }^{1} \mathbf{H} \mathbf{N M R}\left(\mathrm{CDCl}_{3}, 500 \mathrm{MHz}\right) \delta 0.95(\mathrm{t}, J=7.4$ $\mathrm{Hz}, 3 \mathrm{H}), 1.37-1.48(\mathrm{~m}, 2 \mathrm{H}), 1.63-1.72(\mathrm{~m}, 2 \mathrm{H}), 3.81(\mathrm{~s}, 3 \mathrm{H}), 4.19(\mathrm{t}, J=6.7 \mathrm{~Hz}, 2 \mathrm{H}), 6.30(\mathrm{~d}, J=$ 16.0, 1H), 6.85-6.92 (m, 2H), 7.43-7.48 (m, 2H), $7.62(\mathrm{~d}, J=16.0,1 \mathrm{H}) ;{ }^{13} \mathbf{C}$ NMR 13.69, 19.13, $30.73,55.25,64.17,114.20,115.65,127.09,129.60,144.12,161.23,167.35$.

(E)-3-Phenyl-acrylic acid butyl ester. ${ }^{1} \mathbf{H}$ NMR $\left(\mathrm{CDCl}_{3}, 500 \mathrm{MHz}\right) \delta 0.97(\mathrm{t}, J=7.4 \mathrm{~Hz}, 3 \mathrm{H})$, $1.38-1.49(\mathrm{~m}, 2 \mathrm{H}), 1.64-1.73(\mathrm{~m}, 2 \mathrm{H}), 4.21(\mathrm{t}, J=6.7 \mathrm{~Hz}, 2 \mathrm{H}), 6.45(\mathrm{~d}, J=16.0,1 \mathrm{H}), 7.34-7.41(\mathrm{~m}$, $3 \mathrm{H}), 7.49-7.55(\mathrm{~m}, 2 \mathrm{H}), 7.68(\mathrm{~d}, J=16.0,1 \mathrm{H}) ;{ }^{13} \mathbf{C}$ NMR 13.70, 19.15, 30.72, 64.37, 118.22, $127.99,128.81,130.15,134.40,144.49,167.05$.

(E)-3-(4-Bromo-phenyl)-acrylic acid butyl ester. ${ }^{1} \mathbf{H} \mathbf{N M R}\left(\mathrm{CDCl}_{3}, 500 \mathrm{MHz}\right) \delta 0.96(\mathrm{t}, J=7.4$ $\mathrm{Hz}, 3 \mathrm{H}), 1.36-1.48(\mathrm{~m}, 2 \mathrm{H}), 1.61-1.73(\mathrm{~m}, 2 \mathrm{H}), 4.20$ (t, $J=6.7 \mathrm{~Hz}, 2 \mathrm{H}), 6.42$ (d, $J=16.0,1 \mathrm{H}), 7.32-$ $7.41(\mathrm{~m}, 2 \mathrm{H}), 7.47-7.54(\mathrm{~m}, 2 \mathrm{H}), 7.60(\mathrm{~d}, J=16.0,1 \mathrm{H}) ;{ }^{13} \mathbf{C}$ NMR 13.69, 19.12, 30.68, 64.48, $118.92,124.38,129.35,132.04,133.31,143.07,166.74$.

(E)-3-(4-Cyano-phenyl)-acrylic acid butyl ester. ${ }^{1} \mathbf{H}$ NMR $\left(\mathrm{CDCl}_{3}, 500 \mathrm{MHz}\right) \delta 0.95(\mathrm{t}, J=7.4$ $\mathrm{Hz}, 3 \mathrm{H}), 1.35-1.48(\mathrm{~m}, 2 \mathrm{H}), 1.63-1.72(\mathrm{~m}, 2 \mathrm{H}), 4.21$ (t, $J=6.9 \mathrm{~Hz}, 2 \mathrm{H}), 6.51(\mathrm{~d}, J=16.0,1 \mathrm{H}), 7.57-$ 7.70 (m, 5H); ${ }^{13} \mathbf{C}$ NMR 13.66, 19.09, 30.61, 64.77, 113.25, 118.30, 121.80, 128.31, 132.57, $138.68,142.03,166.17$.

(E)-3-p-Tolyl-acrylic acid butyl ester. ${ }^{1} \mathbf{H} \mathbf{N M R}\left(\mathrm{CDCl}_{3}, 500 \mathrm{MHz}\right) \delta 0.97(\mathrm{t}, J=7.4 \mathrm{~Hz}, 3 \mathrm{H})$, 1.38-1.50 (m, 2H), 1.61-1.78 (m, 2H), $2.37(\mathrm{~s}, 3 \mathrm{H}), 4.20(\mathrm{t}, J=6.6 \mathrm{~Hz}, 2 \mathrm{H}), 6.39(\mathrm{~d}, J=16.0 \mathrm{~Hz}$, 1H), 7.12-7.23 (m, 2H), 7.38-7.47 (m, 2H), 7.66 (d, J=16.0 Hz, 1H); ${ }^{13}$ C NMR 13.73, 19.19, $21.44,30.77,64.32,117.17,128.02,129.57,131.72,140.58,144.52,167.28$.

(E)-3-(4-Acetyl-phenyl)-acrylic acid butyl ester. ${ }^{1} \mathbf{H} \mathbf{N M R}\left(\mathrm{CDCl}_{3}, 500 \mathrm{MHz}\right) \delta 0.97(\mathrm{t}, J=7.4$ $\mathrm{Hz}, 3 \mathrm{H}), 1.34-1.52(\mathrm{~m}, 2 \mathrm{H}), 1.62-1.76(\mathrm{~m}, 2 \mathrm{H}), 2.61(\mathrm{~s}, 3 \mathrm{H}), 4.22(\mathrm{t}, J=6.6 \mathrm{~Hz}, 2 \mathrm{H}), 6.53(\mathrm{~d}, J=$ $16.0 \mathrm{~Hz}, 1 \mathrm{H}), 7.54-7.64(\mathrm{~m}, 2 \mathrm{H}), 7.69(\mathrm{~d}, J=16.0 \mathrm{~Hz}, 1 \mathrm{H}), 7.92-8.01(\mathrm{~m}, 2 \mathrm{H}) ;{ }^{13} \mathrm{C}$ NMR 13.71 , $19.15,26.66,30.67,64.66,120.82,128.09,128.82$, 137.93, 138.78, 142.94, 166.57, 197.30.

(E)-4-(2-Butoxycarbonyl-vinyl)-benzoic acid butyl ester. Pale yellow oil, ${ }^{\mathbf{1}} \mathbf{H} \mathbf{N M R}\left(\mathrm{CDCl}_{3}, 500\right.$ $\mathrm{MHz}) \delta 0.97$ (two triplets partially overlapped, $J=7.4 \mathrm{~Hz}, 6 \mathrm{H}), 1.36-1.54(\mathrm{~m}, 4 \mathrm{H}), 1.64-1.80$ (m, $4 \mathrm{H}), 4.21(\mathrm{t}, J=6.6 \mathrm{~Hz}, 2 \mathrm{H}), 4.32(\mathrm{t}, J=6.6 \mathrm{~Hz}, 2 \mathrm{H}), 6.51(\mathrm{~d}, J=16.0 \mathrm{~Hz}, 1 \mathrm{H}), 7.54-7.62(\mathrm{~m}, 2 \mathrm{H})$, $7.68(\mathrm{~d}, J=16.0 \mathrm{~Hz}, 1 \mathrm{H}), 8.00-8.08(\mathrm{~m}, 2 \mathrm{H}) ;{ }^{13} \mathrm{C}$ NMR 13.72, 19.1, 19.22, 30.69, 64.60, 65.03, 120.55, 127.81, 130.00, 131.67, 138.53, 143.13, 165.99, 166.60; IR liquid film v 2960, 2873, 1717, 
1639, 1569, 1456, 1412, 1386, 1277, 1203, 1172, 1105, 1018, 849, $774 \mathrm{~cm}^{-1}$; MS (m/z) $304\left(\mathrm{M}^{+}, 8\right)$, 248 (15), 231 (40); 192 (100), 175 (34), 147 (31), 102 (16), 91 (12), 77 (12), 57 (40), 56 (46), 41 (77); HRMS calcd. for $\mathrm{C}_{18} \mathrm{H}_{24} \mathrm{O}_{4} \quad 304.1675$, found 304.1655. 

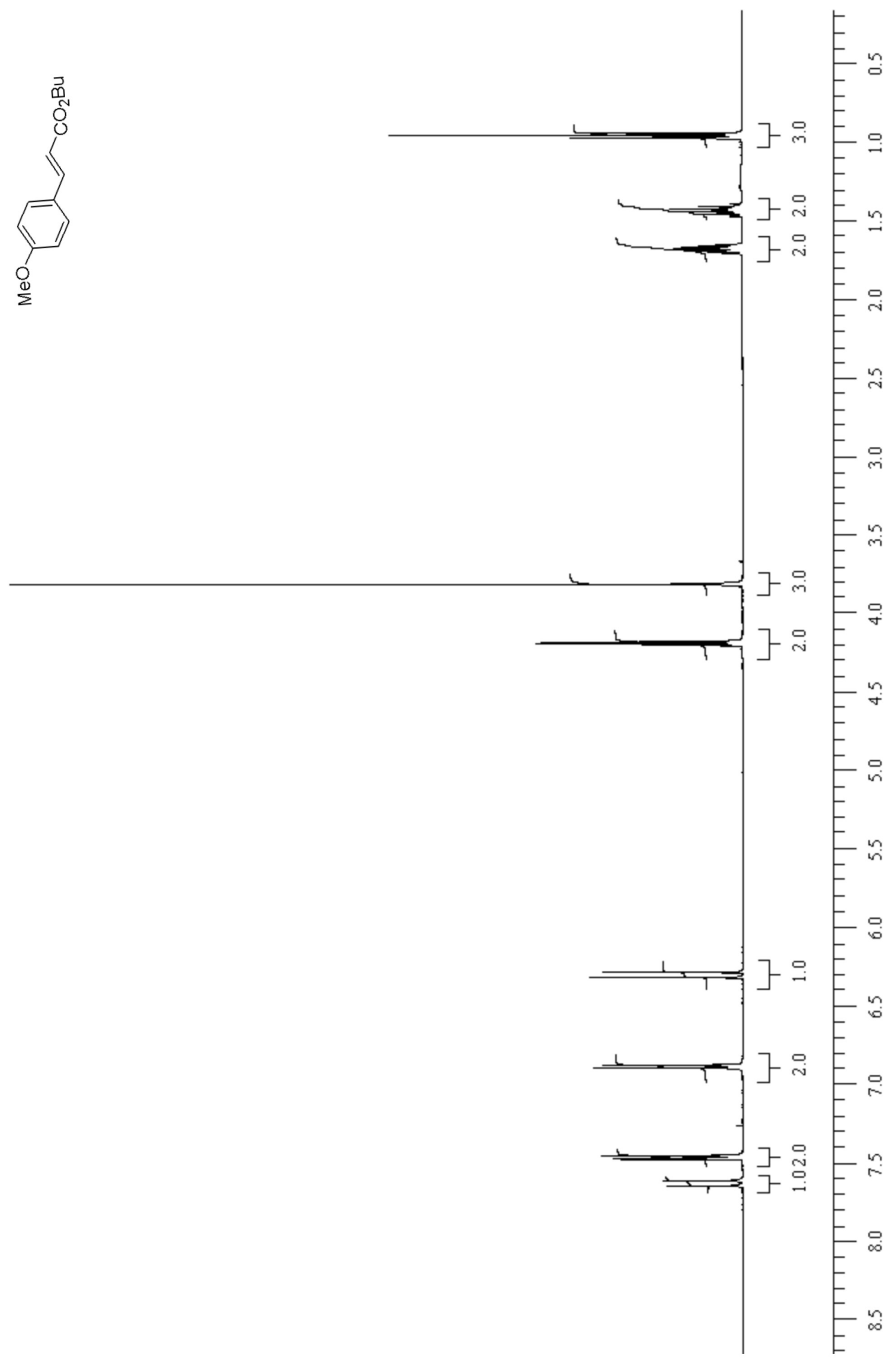

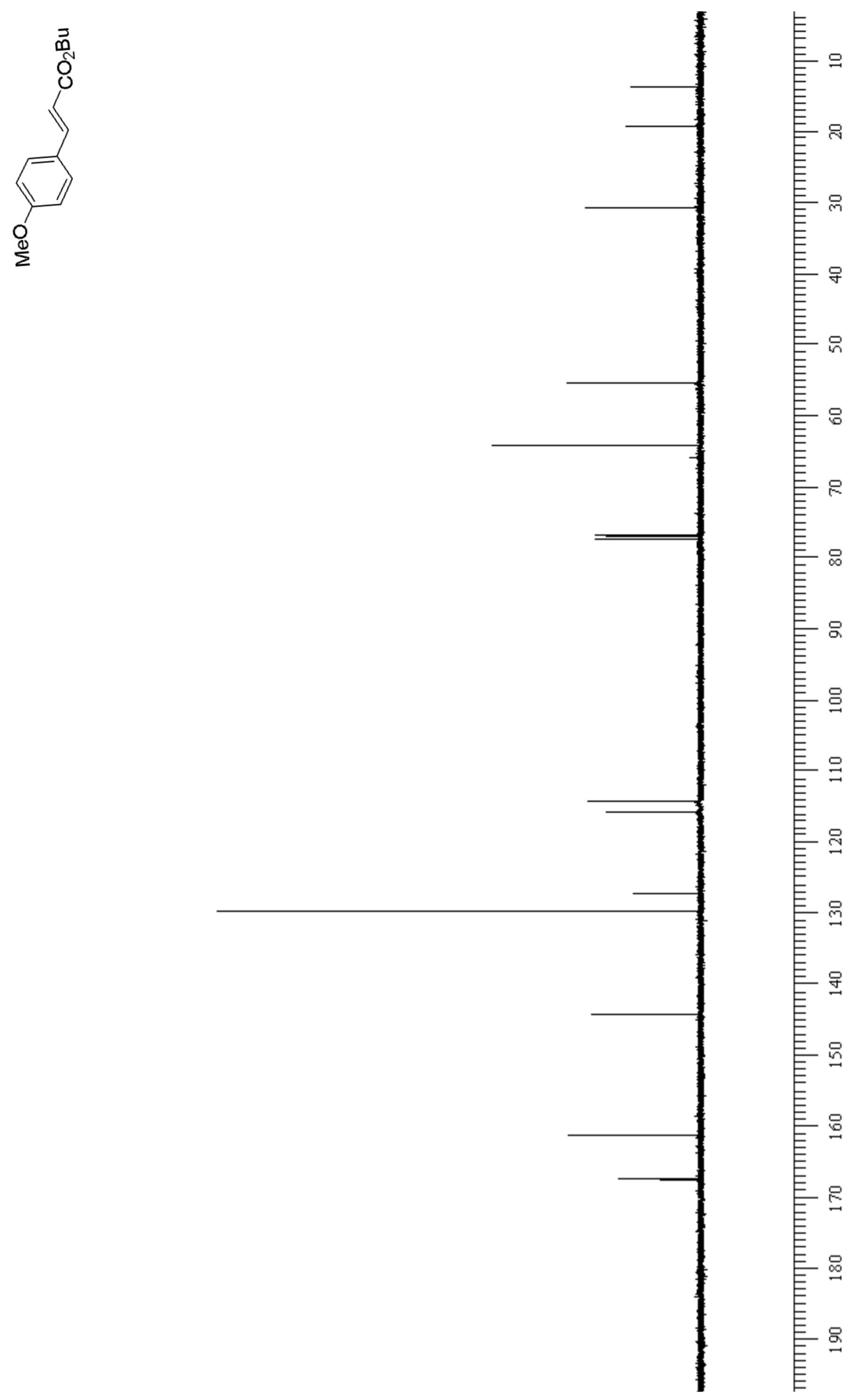

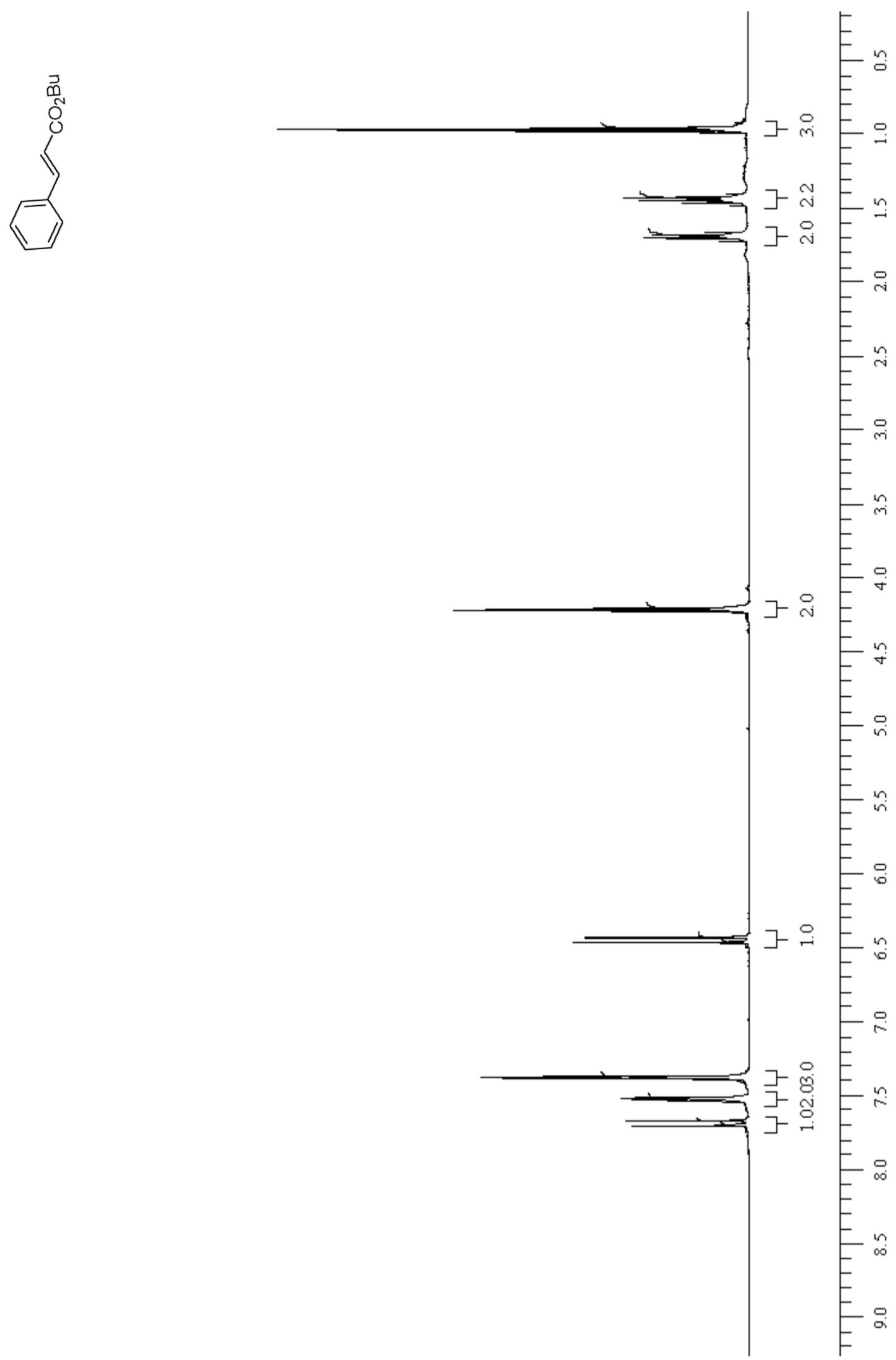

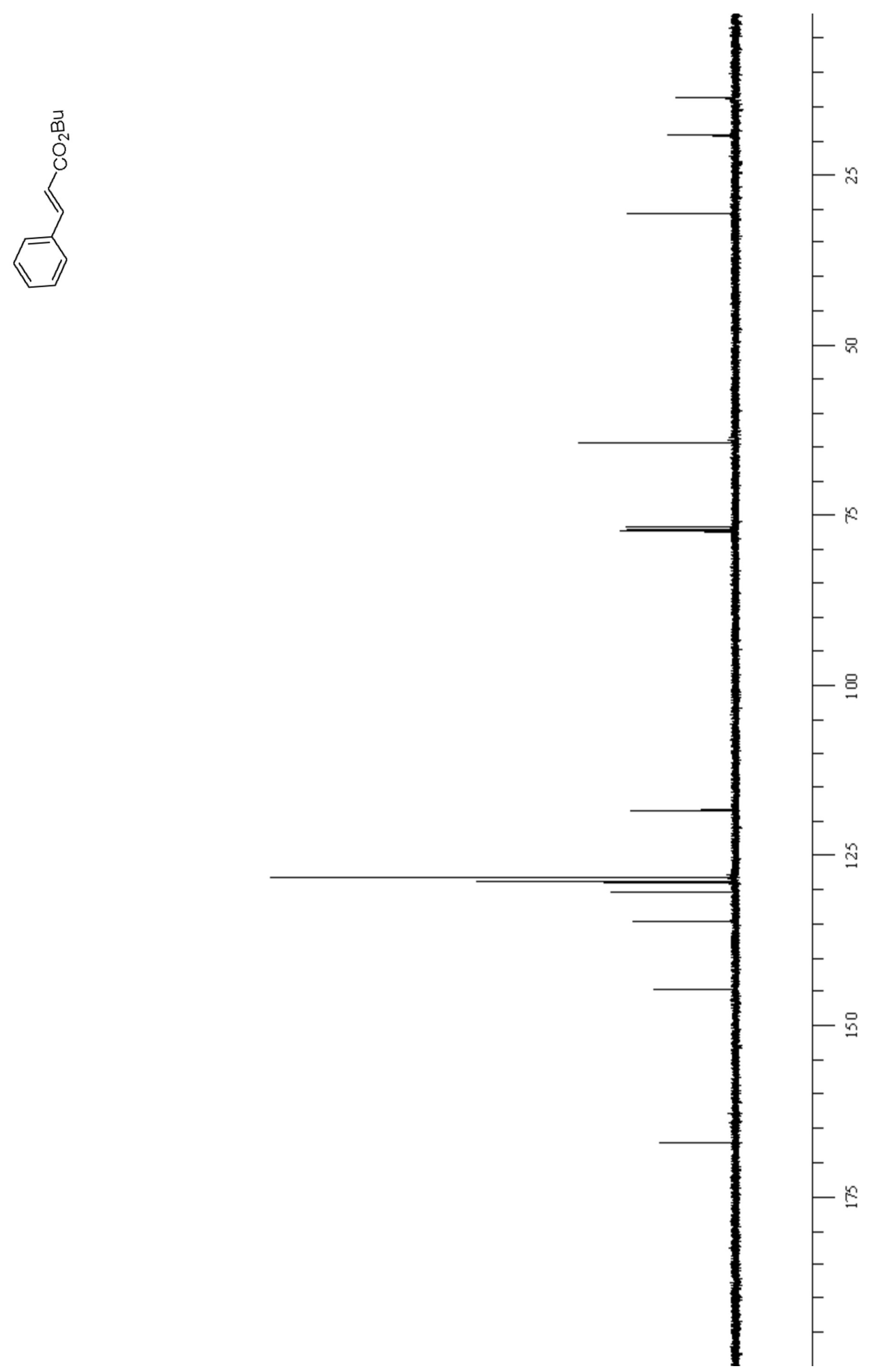


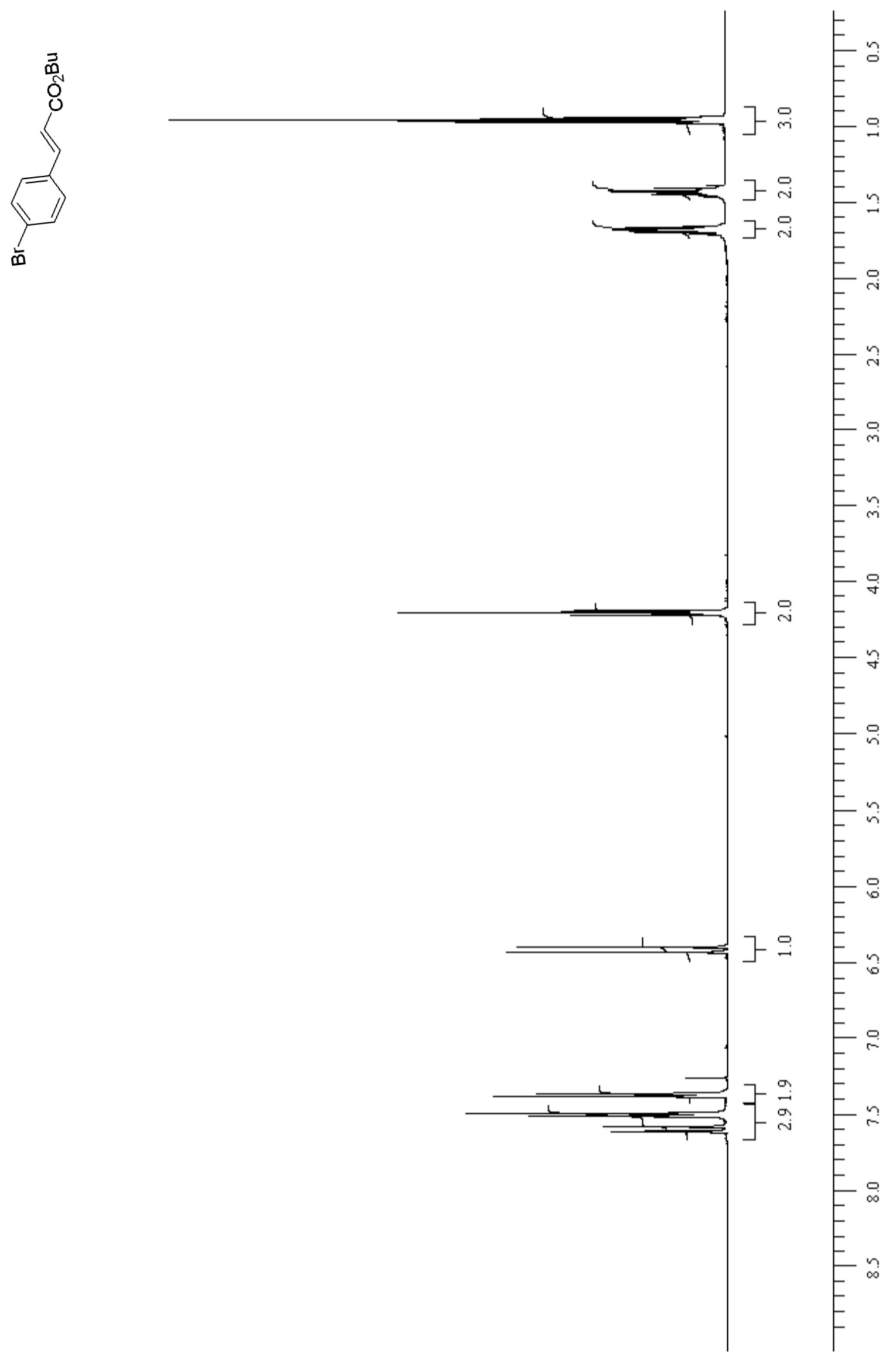



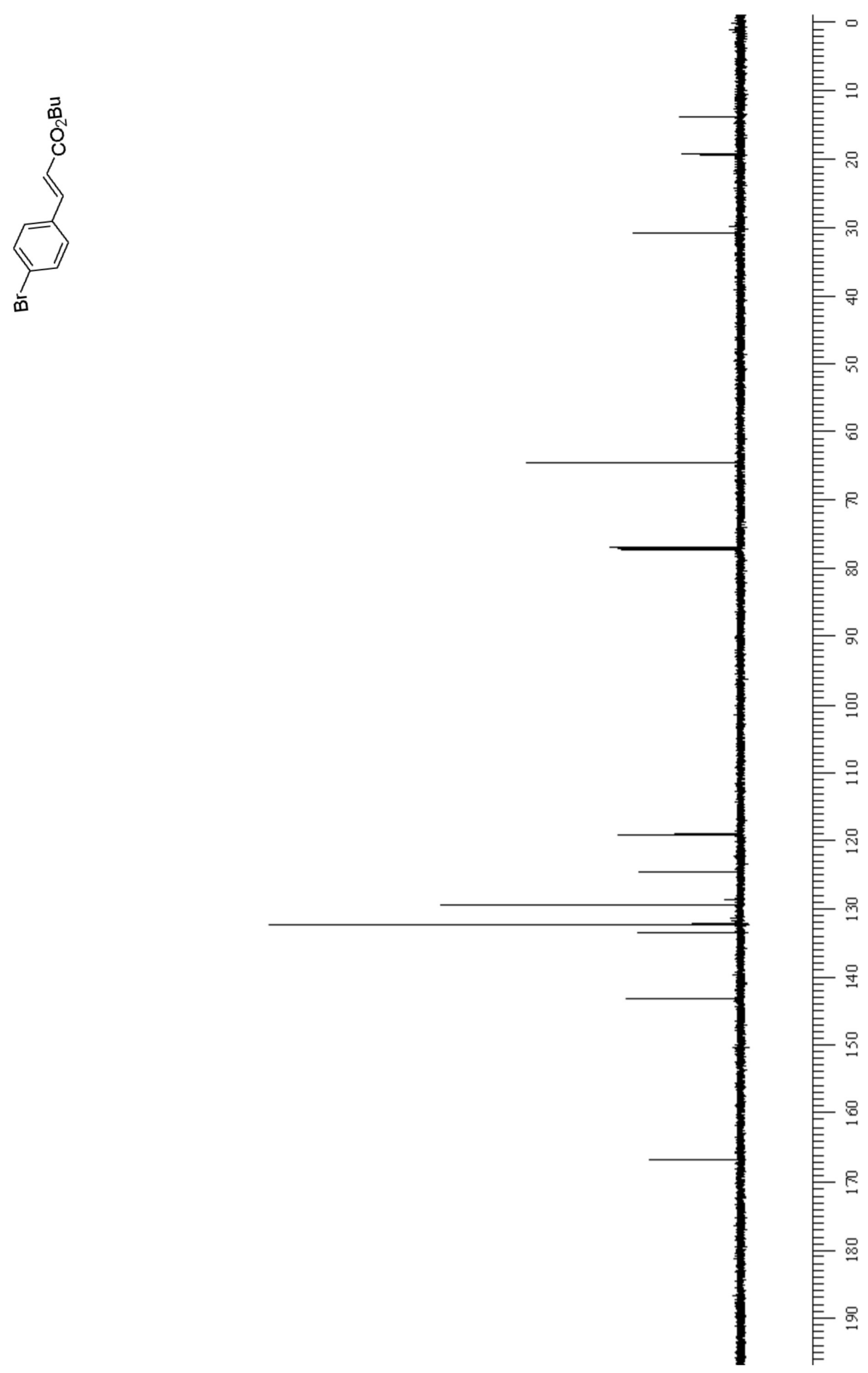

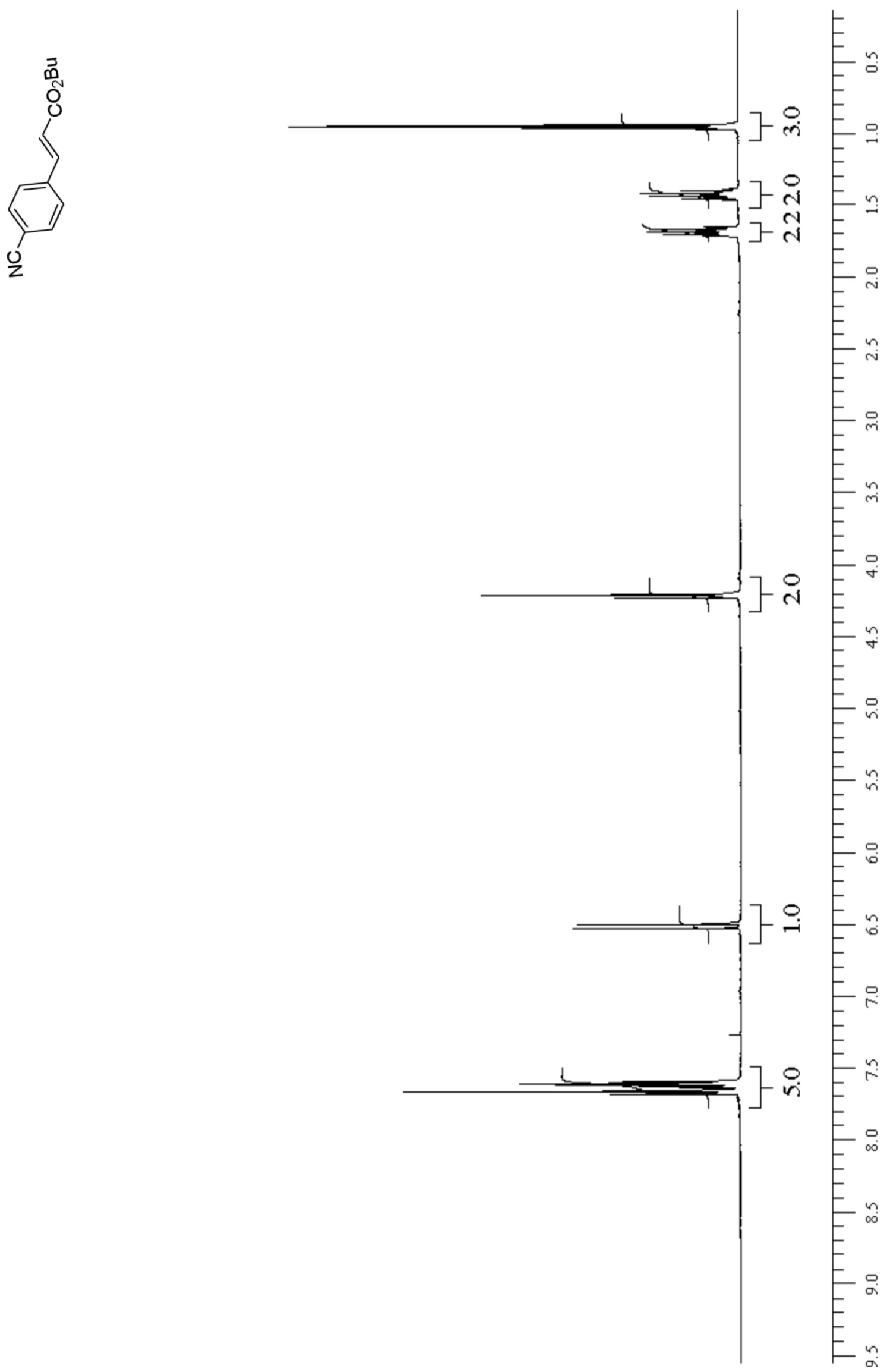


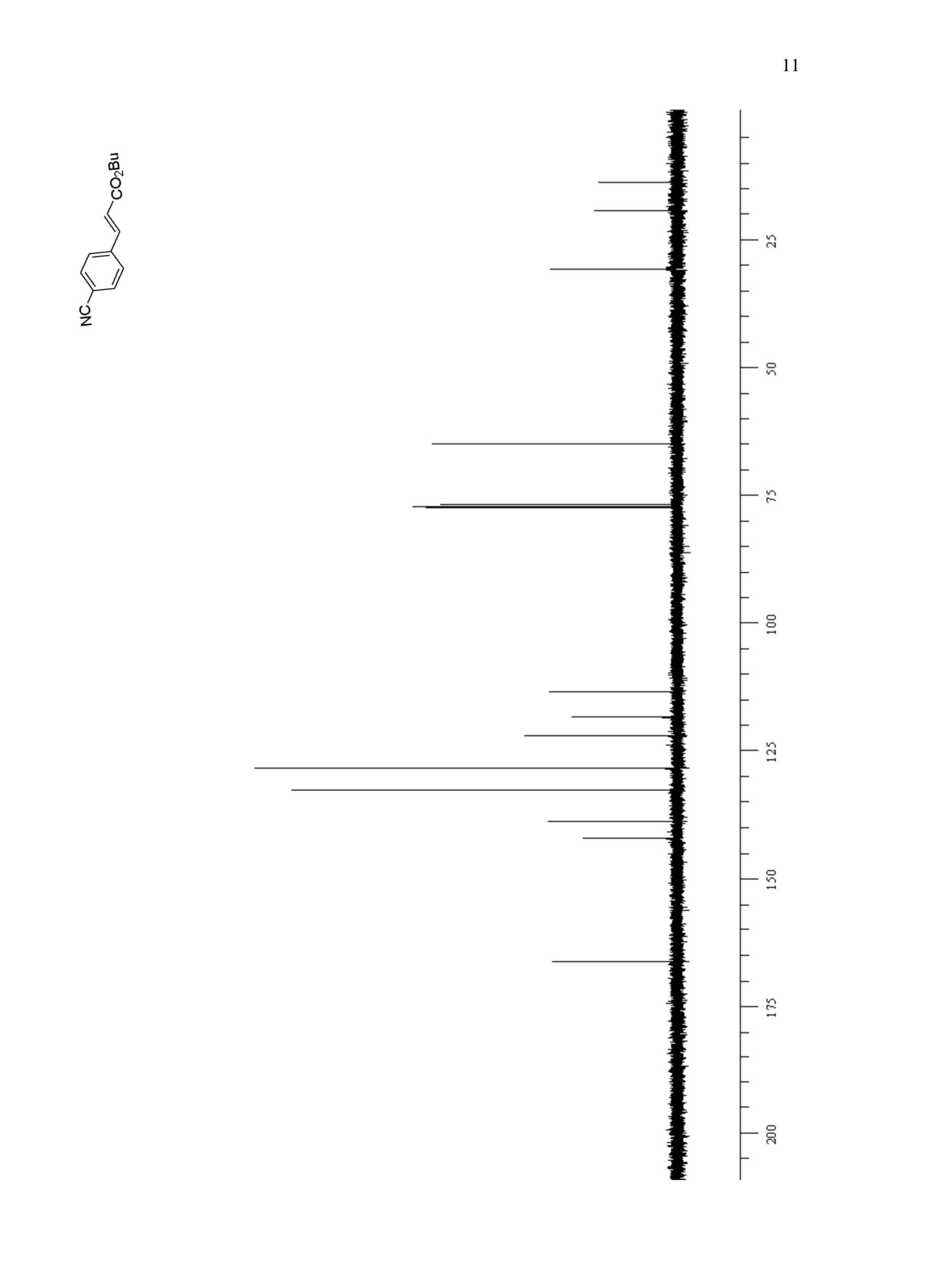




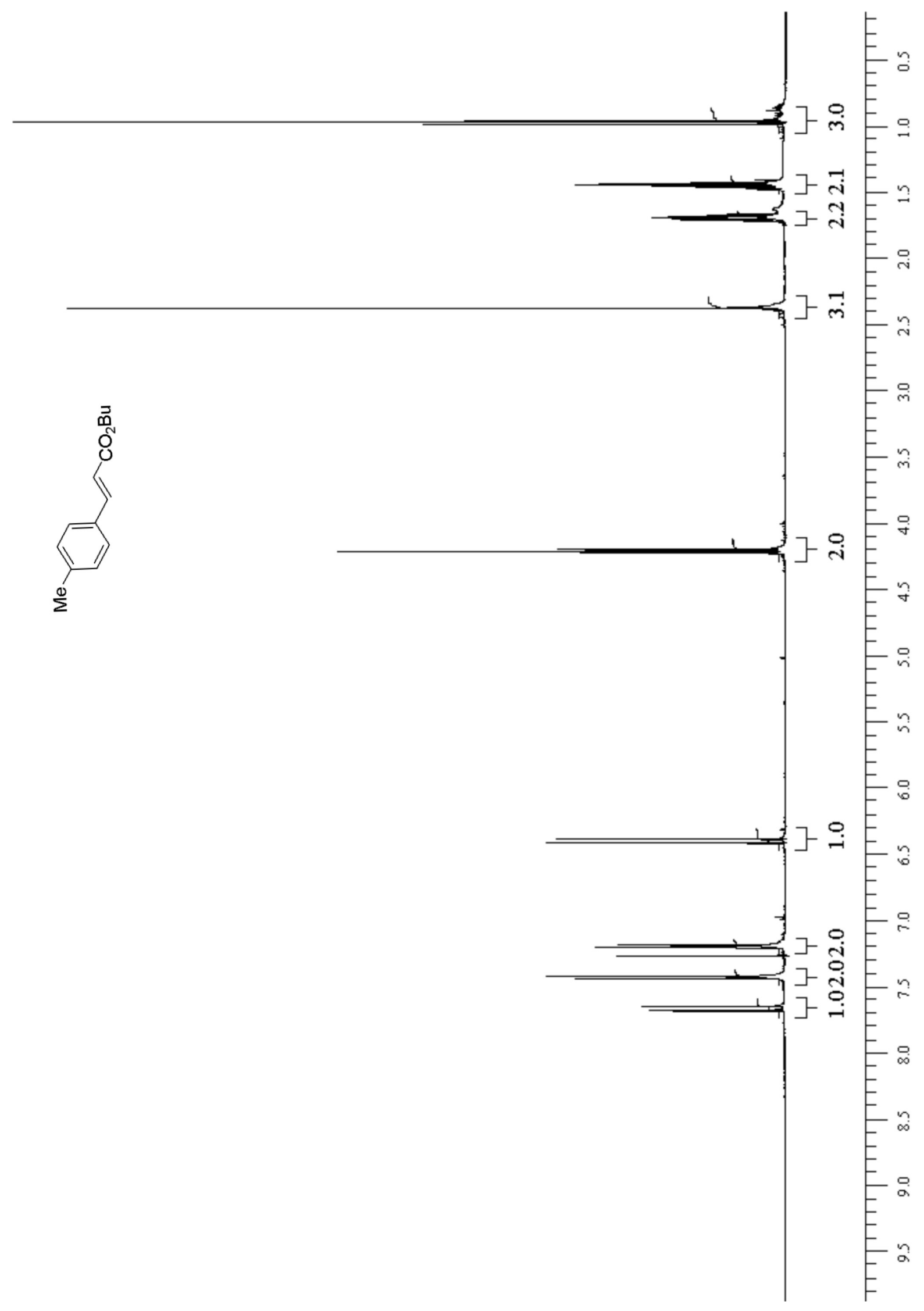



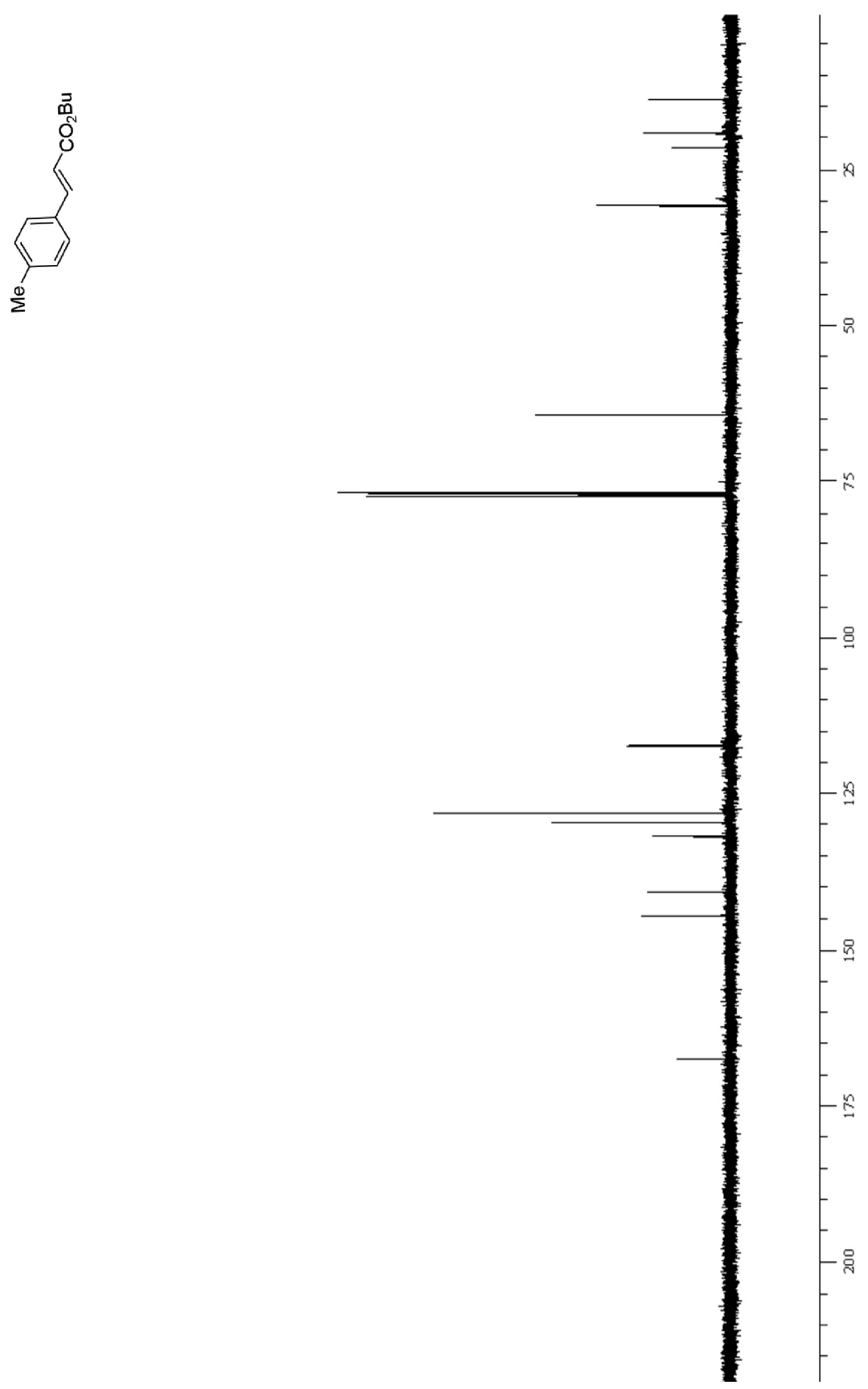

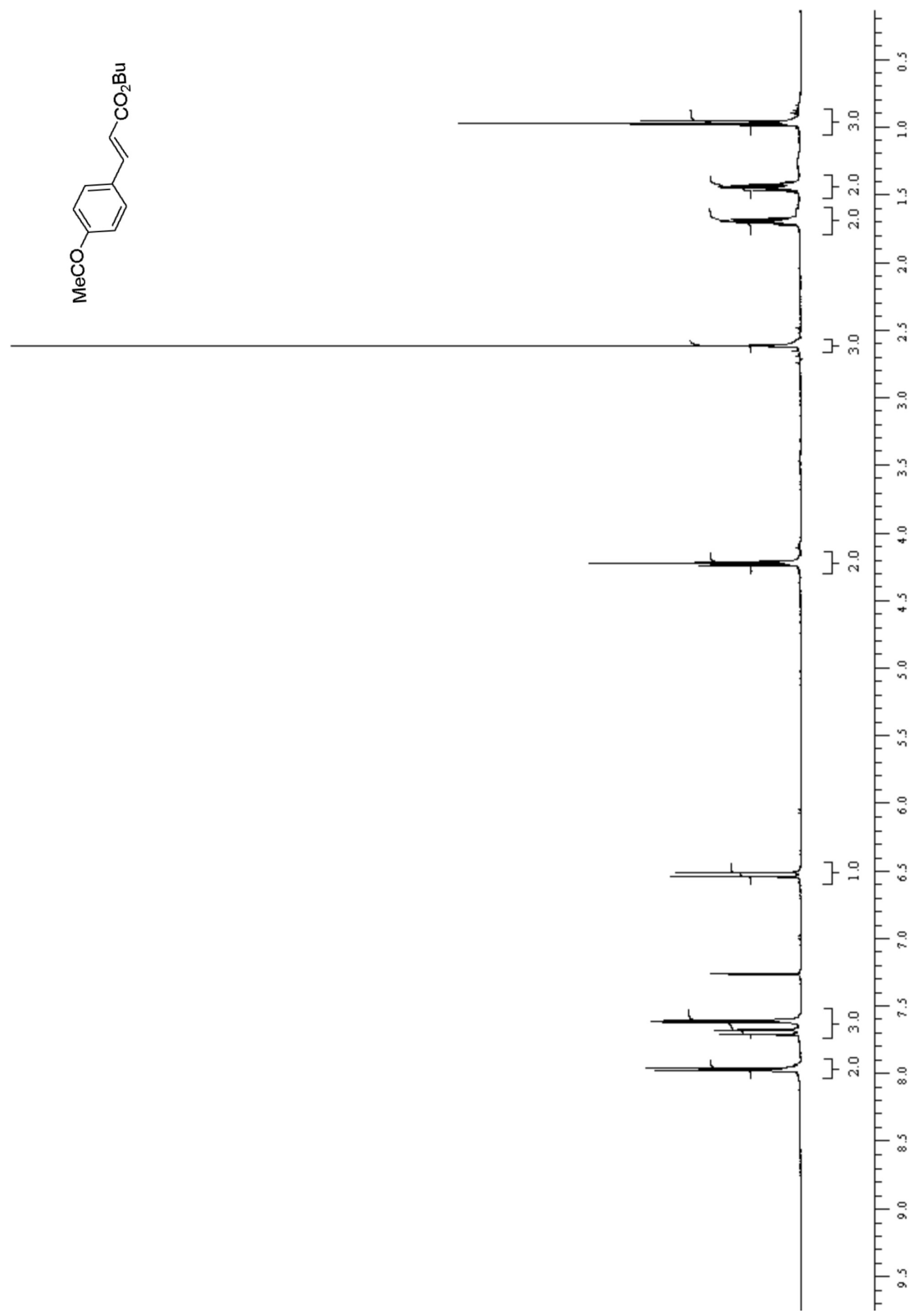


$$
1
$$



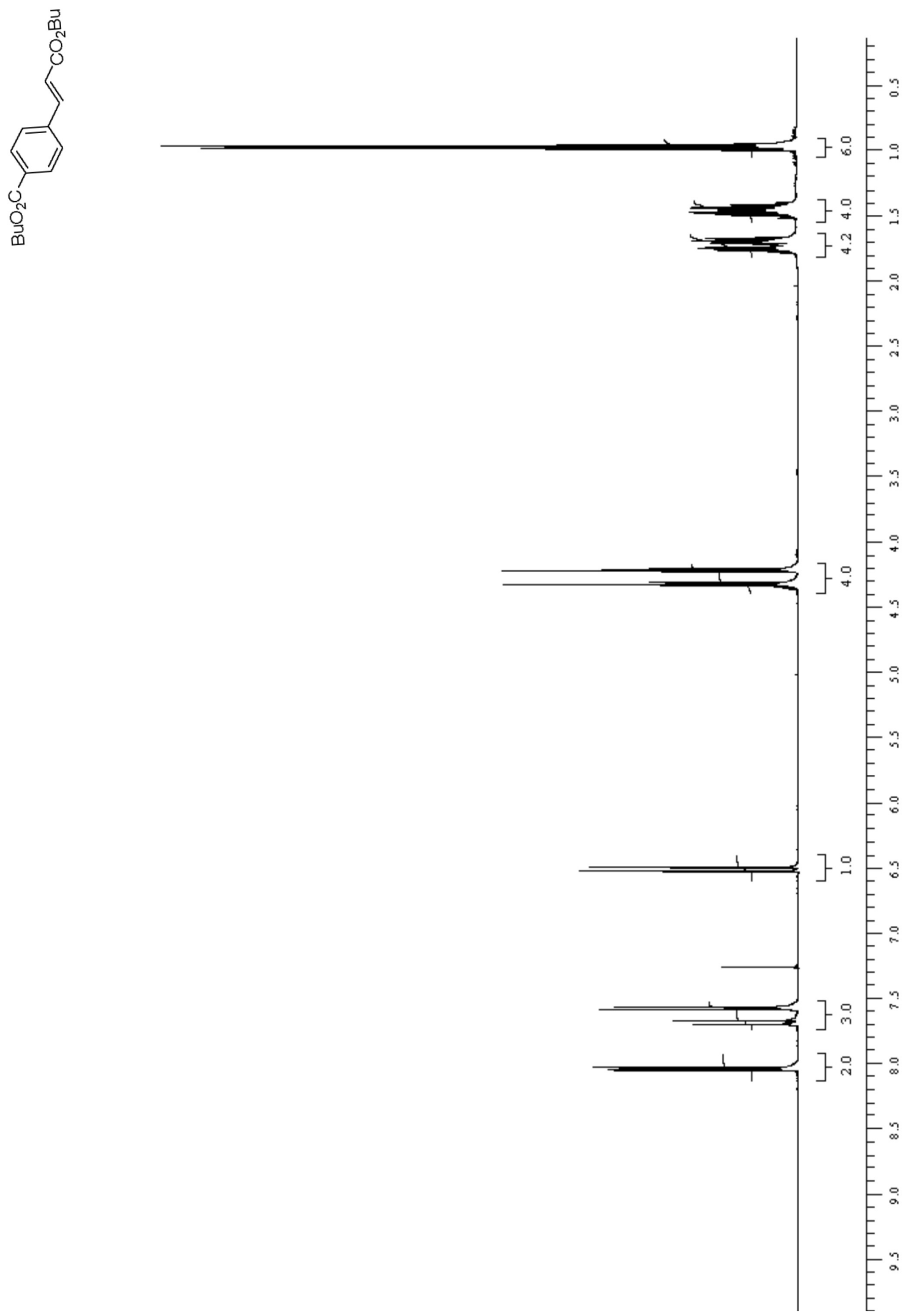

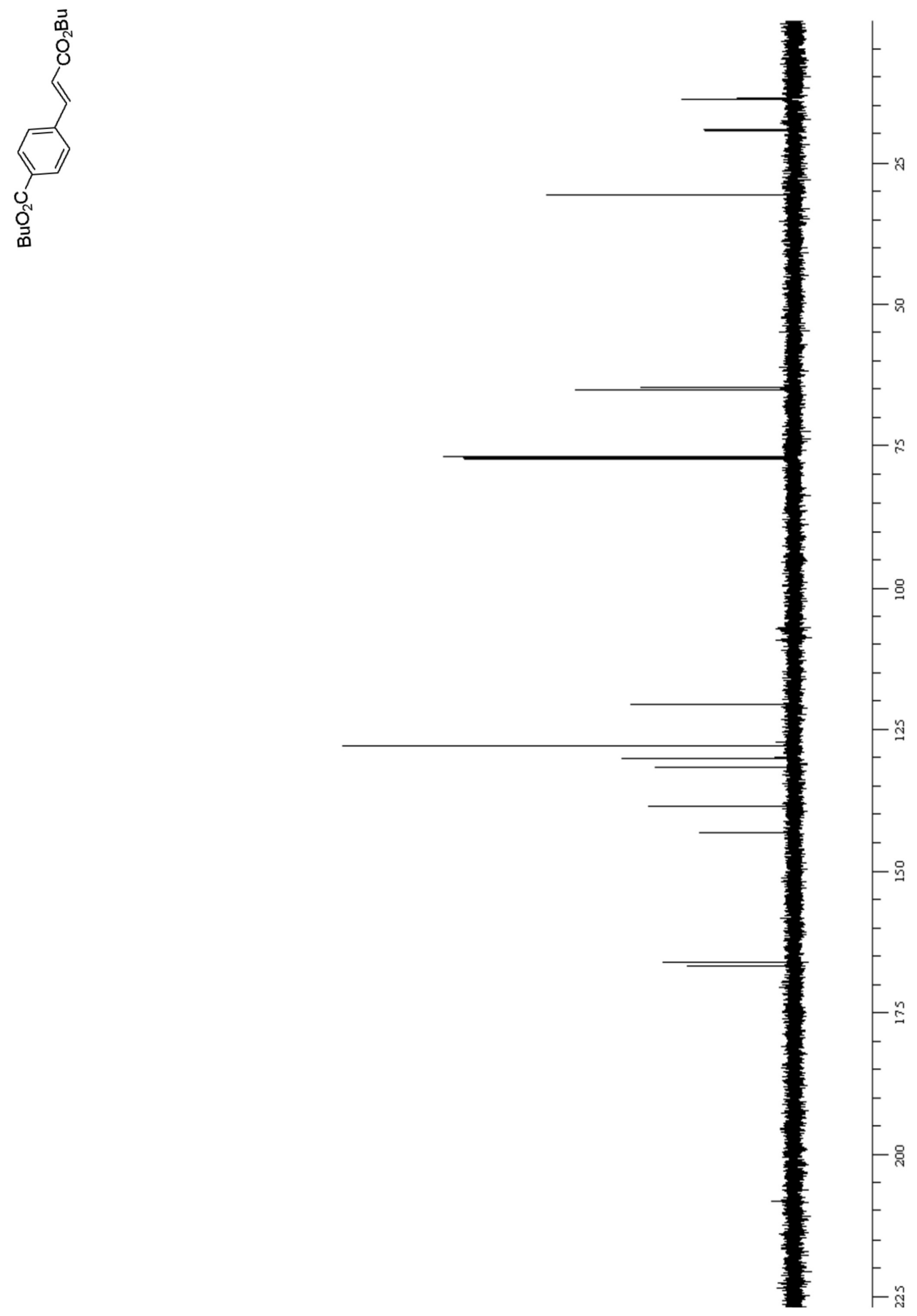